\title{
Molecular Imaging of the Glomerulus via Mesangial Cell Uptake of Radiolabeled Tilmanocept
}

\author{
Zhengtao Qin ${ }^{1,2}$, Carl K. Hoh ${ }^{1,2}$, Emilia S. Olson ${ }^{1,2}$, Amin Haghighat Jahromi ${ }^{1,2}$, David J. Hall ${ }^{1,2}$, Christopher V. Barback ${ }^{1,2}$, \\ Young-Hyun $\mathrm{You}^{3,4}$, Motoko Yanagita ${ }^{5}, \mathrm{Kumar}_{\text {Sharma }}{ }^{3,4}$, and David R. Vera ${ }^{1,2}$ \\ ${ }^{I}$ Department of Radiology, University of California, San Diego, La Jolla, California; ${ }^{2}$ In Vivo Cancer and Molecular Imaging \\ Program, University of California, San Diego, La Jolla, California; ${ }^{3}$ Center for Renal Translational Medicine, University of \\ California, San Diego, La Jolla, California; ${ }^{4}$ Department of Medicine, Division of Nephrology, University of California, San Diego, \\ La Jolla, California; and ${ }^{5}$ Department of Nephology, Kyoto University, Kyoto, Japan
}

An unmet need for the clinical management of chronic kidney disease is a predictive tool of kidney function during the first decade of the disease, when there is silent loss of glomerular function. The objective of this study was to demonstrate receptormediated binding of tilmanocept to CD206 within the kidney and provide evidence of kinetic sensitivity of this binding to renal function. Methods: Rats were positioned in a PET scanner with the liver and kidneys within the field of view. After an intravenous injection of ${ }^{68} \mathrm{Ga}-I R D y e 800$-tilmanocept, using 1 of 2 scaled molar doses $(0.02 \mathrm{nmol} / \mathrm{g}, n=5$; or $0.10 \mathrm{nmol} / \mathrm{g}, n=5)$, or coinjection $(n=3)$ of ${ }^{68} \mathrm{Ga}-I R D y e 800$-tilmanocept $(0.10 \mathrm{nmol} / \mathrm{g})$ and unlabeled tilmanocept $(5.0 \mathrm{nmol} / \mathrm{g})$, or a negative control, ${ }^{68} \mathrm{Ga}-\mathrm{IRDye} 800-$ DTPA-galactosyl-dextran $(0.02 \mathrm{nmol} / \mathrm{g}, n=5)$, each animal was imaged for 20 min followed by a whole-body scan. Frozen kidney sections were stained for podocytes and CD206 using immunofluorescence. Molecular imaging of diabetic $d b / d b$ mice ( $4.9 \mathrm{wk}, n=$ $6 ; 7.3 \mathrm{wk}, n=4 ; 13.3 \mathrm{wk}, n=6)$ and nondiabetic $\mathrm{db} / \mathrm{m}$ mice $(n=6)$ was performed with fluorescence-labeled $99 \mathrm{~m}$ Tc-tilmanocept (18.5 MBq, $2.6 \mathrm{nmol}$ ). Thirty minutes after injection, blood, liver, kidneys, and urine were assayed for radioactivity. Renal time-activity curves were generated. Results: Rat PET whole-body images and time-activity curves of ${ }^{68} \mathrm{Ga}$-IRDye800-tilmanocept demonstrated receptor-mediated renal accumulation with evidence of glomerular uptake. Activity within the renal cortex persisted during the 40-min study. Histologic examination demonstrated colocalization of CD206 and IRDye800-tilmanocept within the glomerulus. The glomerular accumulation of the coinjection and the negative control studies were significantly less than the CD206-targeted agent. The $d b / d b$ mice displayed a multiphasic renal time-activity curve with high urinary bladder accumulation; the nondiabetic mice exhibited renal uptake curves dominated by a single phase with low bladder accumulation. Conclusion: This study demonstrated receptor-mediated binding to the glomerular mesangial cells and kinetic sensitivity of tilmanocept to chronic renal disease. Given the role of mesangial cells during the progression of diabetic nephropathy, PET or SPECT renal imaging with radiolabeled tilmanocept may provide a noninvasive quantitative assessment of glomerular function.

\footnotetext{
Received Nov. 26, 2018; revision accepted Jan. 24, 2019.

For correspondence or reprints contact: David R. Vera, Department of Radiology, University of California, San Diego, 3855 Health Sciences Dr., \#0819, La Jolla, CA 92093.

E-mail: dvera@ucsd.edu

Published online Feb. 22, 2019.

COPYRIGHT (C 2019 by the Society of Nuclear Medicine and Molecular Imaging.
}

Key Words: kidney imaging; ${ }^{99 m T c-t i l m a n o c e p t ; ~}{ }^{88}$ Ga-tilmanocept; mesangial cells

J Nucl Med 2019; 60:1325-1332

DOI: 10.2967/jnumed.118.223727

$\tau$ n 2017, the International Diabetes Federation estimated that the worldwide prevalence of diabetes would increase from 415 million persons in 2015 to 642 million in 2040 (1). Approximately $40 \%$ of persons with diabetes develop diabetic nephropathy as manifested by albuminuria or impaired glomerular filtration rate (2). As diabetic kidney disease progresses over 2-3 decades, it is critical to have a noninvasive measure that monitors early disease activity since markers such as albuminuria may often be transient and reductions in the estimates of glomerular filtration rate would already indicate more advanced disease (3). Consequently, the detection of renal dysfunction within the first decade of diabetic nephropathy is an unmet clinical need (4).

We propose external imaging of mesangial cell function as a biomarker for diabetic nephropathy. Our reasoning is based on the following. Mesangial cell matrix expansion is a histologic hallmark of diabetic nephropathy (5), which precedes the reduction of a patient's glomerular filtration rate (6). Additionally, all of the clinical manifestations of diabetic nephropathy are highly correlated with mesangial matrix expansion $(7,8)$. Lastly, there is evidence the mesangial expansion is reversable with therapy (9). There currently does not exist an imaging, serum, or urine biomarker that is sensitive to mesangial cell function (10).

We propose molecular imaging of mesangial cell function by targeting a radiopharmaceutical to CD206, which resides on the surface of mesangial cells (11). The imaging agent would be based on the design strategy for Tc-tilmanocept (12). ${ }^{99 \mathrm{~m} T c-t i l m a n o c e p t,}$ which has regulatory approvals in the United States and Europe for sentinel lymph node mapping, uses a diethylenetriaminepentaacetic acid (DTPA)-mannosyl conjugate of dextran (Fig. 1) and offers several advantages for the design of a renal imaging agent. The molecular weight of the dextran backbone can be adjusted to match the clinical requirements (13), and the number of receptor substrates per dextran can be altered to optimize receptor affinity (14). Tilmanocept can be labeled with ${ }^{68} \mathrm{Ga}$ for PET imaging (15). During the design process, dextran conjugates such as tilmanocept can be labeled with a fluorophore without the loss of receptor affinity (16). Additionally, chemical 


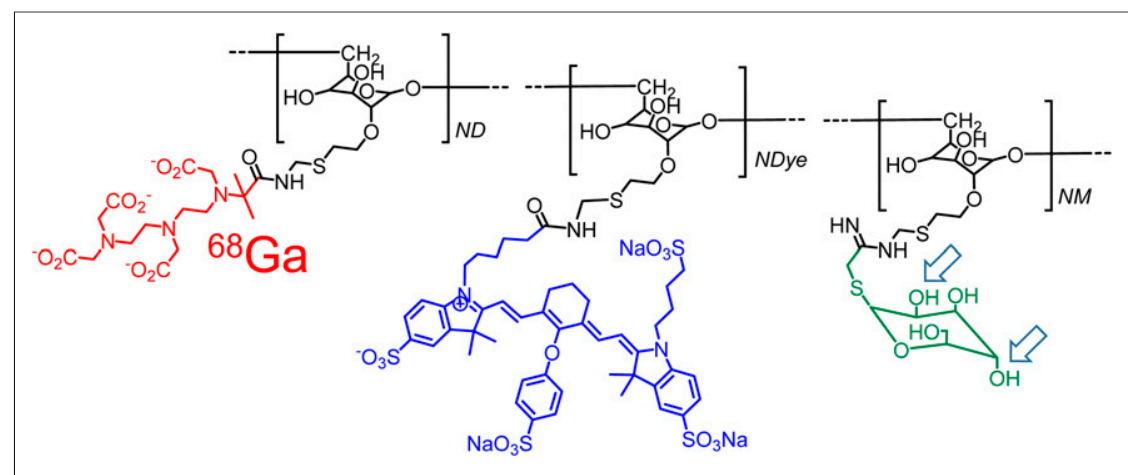

FIGURE 1. Bimodal molecular imaging agent, ${ }^{88} \mathrm{Ga}$-labeled IRDye800-tilmanocept, consists of modified dextran backbone (black), which carries CD206 receptor substrate, mannose (green); metal chelator, DTPA (red); and fluorescent tag, IRDye800CW (blue). Isomeric change of hydroxyls at $\mathrm{C} 2$ and $\mathrm{C} 4$ carbons of the receptor substrate (arrows) produces glycoconjugate that binds only to asialoglycoprotein receptor, which is specific to hepatocytes.

isomers of the receptor substrate can be used to produce a negative control with completely different receptor specificity but similar hydrodynamic properties (Fig. 1).

In this work, we present 5 independent experiments that demonstrate glomerular CD206 accumulation of tilmanocept and sensitivity to diabetic nephropathy. The first 3 experiments used PET imaging of healthy rats to obtain adequate spatial resolution of the renal cortex. We used a galactosyl-dextran conjugate as a negative control. We used 2 different in vivo receptor saturation methods to demonstrate changes in renal PET time-activity data. We tested the histologic colocalization of fluorescent tilmanocept with a histoimmunofluorescent marker for CD206. Lastly, we tested the kinetic sensitivity to mesangial cell matrix expansion by comparing the renal time-activity curves of nondiabetic $\mathrm{db} / \mathrm{m}$ and diabetic $d b / d b$ mice; the latter animal model exhibits mesangial cell matrix expansion. We conclude with a discussion of the chemical modifications of tilmanocept that will optimize a molecular imaging protocol for staging a patient with early diabetic nephropathy.

\section{MATERIALS AND METHODS}

\section{Reagents and Animals}

Tilmanocept, DTPA-mannosyl-dextran, and DTPA-dextran (12) were provided by Navidea Biopharmaceuticals. IRDye800CW-NHSester was purchased from LI-COR Biosciences, and Cy5-NHS-ester was purchased from GE Healthcare. Female diabetic C57BLKS/J LepR $^{\mathrm{db}} / \operatorname{LepR}^{\mathrm{db}}$ mice $(d b / d b)$ and female nondiabetic C57BLKS/J LepR $^{\mathrm{db}}(\mathrm{db} / \mathrm{m})$ were purchased from Jackson Laboratories. This study was approved by the University of California, San Diego, Institutional Animal Care and Use Committee.

\section{Bimodal Radiopharmaceuticals}

DTPA-galactosyl-dextran was synthesized (12) using cyanomethyl$2,3,4,6$-tetra- $O$-acetyl-1-thio- $\beta$-D-galactopyranoside (17). IRDye $800 \mathrm{CW}$ or Cy5 was covalently attached to tilmanocept or DTPA-galactosyl-dextran as previously described (18). The average fluorophore, chelator, carbohydrate, and amine densities for each dextran conjugate were measured by nuclear magnetic resonance (18) and were approximately 2 IRDye $800 \mathrm{CW}$ or Cy5 molecules, 5 DTPAs, 15 thioglycosides, and 0 amino groups per dextran.

IRDye800-tilmanocept or IRDye800-DTPA-galactosyl-dextran was radiolabeled with ${ }^{68} \mathrm{Ga}$ as previously described (19). Cy5-tilmanocept was radiolabeled with ${ }^{99 \mathrm{~m}} \mathrm{Tc}$ using tin reduction (12). Quality control of each fluorescent radiopharmaceutical was performed by instant thin-layer chromatography (19). The radiochemical and optical purities of each injectate exceeded $96 \%$.

\section{PET Imaging of Healthy Rats: Demonstration of Glomerular CD206 Accumulation}

PET imaging of ${ }^{68} \mathrm{Ga}-$ labeled fluorescenttilmanocept was performed using 2 scaled molar doses. Five rats $(154-170 \mathrm{~g})$ were injected with a $0.02 \mathrm{nmol} / \mathrm{g}$ concentration of ${ }^{68} \mathrm{Ga}$-IRDye800-tilmanocept (7.4-13.3 MBq), and 5 rats $(171-214 \mathrm{~g})$ were imaged $(6.3-$ $16.3 \mathrm{MBq}$ ) with a scaled molar dose of 0.10 nmol per gram of body weight. Additionally, 5 rats $(221-252 \mathrm{~g})$ were imaged with ${ }^{68} \mathrm{Ga}$ IRDye800-galactosyl-dextran $(0.02 \mathrm{nmol} / \mathrm{g}$, 7.0-13.3 MBq), and 3 rats $(166-180 \mathrm{~g})$ were imaged after a coinjection of ${ }^{68} \mathrm{Ga}$-IRDye800tilmanocept (0.1 nmol/g, 5.8-4.7 MBq) and unlabeled tilmanocept (5.0 nmol/g). After positioning both kidneys within the field of view of a high-resolution PET scanner (Vista DR; GE Healthcare), we performed a 20-min dynamic scan immediately followed by a whole-body imaging study. At $40 \mathrm{~min}$ after injection, each rat was euthanized by $\mathrm{CO}_{2}$ asphyxiation. Both kidneys, the entire liver, and the urinary bladder were excised, rinsed, weighed, assayed for radioactivity ( $\gamma-8000$; Beckman Instruments [400-600 keV]), bisected, placed in embedding medium, and stored at $-80^{\circ} \mathrm{C}$.

The dynamic scan was reconstructed into cross-sections with 30-s time intervals. Ten contiguous coronal cross-sections from each kidney and liver were used to generate decay-corrected time-activity curves. These curves were scaled to percentage injected dose per gram $(\% \mathrm{ID} / \mathrm{g})$ based on measurements of each kidney and liver obtained at $40 \mathrm{~min}$ after injection. The whole-body images were reconstructed into transaxial cross-sections and reformatted into maximum-intensity-projection images (20).

\section{Immunohistochemistry: Demonstration of CD206 Targeting}

The bisected rat kidneys were cut into contiguous $5-\mu \mathrm{m}$ cryostat sections, stored at $-80^{\circ} \mathrm{C}$, and dried at room temperature before immunofluorescent staining. All incubation solutions contained $40 \mathrm{mM}$ divalent cations. After fixation, the slides were washed and incubated in phosphate-buffered saline containing $0.5 \%$ Triton X-100. This was followed by incubation in phosphate-buffered saline containing $1 \%$ bovine serum albumin and $0.02 \%$ Triton $\mathrm{X}-100$. The 2 primary antibodies, antimannose receptor antibody (ab-64693; ABCAM) and antipodocyte antibody (sc-22296, Santa Cruz Biotechnology), were incubated overnight at $4^{\circ} \mathrm{C}$. After a similar incubation with the secondary antibodies bearing Alexa Fluor 488 or Alexa Fluor 647, the slides were washed, dried, mounted, and stored in the dark at $-20^{\circ} \mathrm{C}$.

The histologic sections were examined under a fluorescence microscope (Nikon E600). Three filter sets were used: excitation, $470 \mathrm{~nm}$, and emission, $525 \mathrm{~nm}$, which represented the podocyte stain; excitation, $620 \mathrm{~nm}$, and emission, $700 \mathrm{~nm}$, which represented the CD206 stain; and excitation, $747 \mathrm{~nm}$; emission, $776 \mathrm{~nm}$, for IRDye800-tilmanocept or IRDye800-DTPA-galactosyl-dextran.

\section{y-Imaging of Mice: Demonstration of Sensitivity to Chronic Renal Disease}

Male diabetic $d b / d b$ mice were imaged at $4.9(n=6), 7.3(n=5)$, and $13.3(n=6)$ weeks of age. Additionally, 4.1-wk-old male nondiabetic $\mathrm{db} / \mathrm{m}$ mice $(n=6)$ were imaged. After weighing, each animal was anesthetized ( $2 \%$ isoflurane, $200 \mathrm{~mL} / \mathrm{min}$ flow of $\mathrm{O}_{2}$ ) and positioned (ventral view) atop a high-resolution $\gamma$-imager $(\gamma$ Imager; BioSpace) 


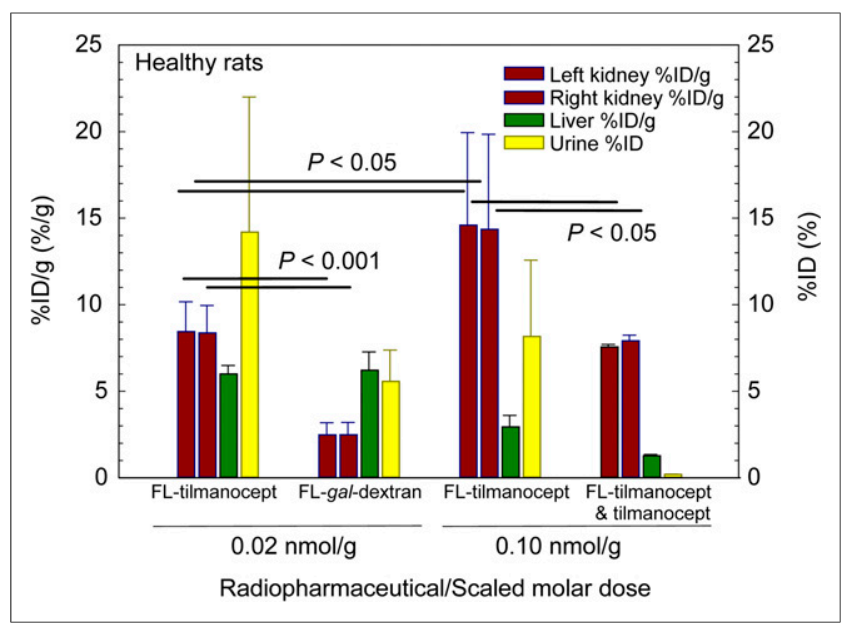

FIGURE 2. In vivo demonstration of receptor-mediated binding by tilmanocept. Kidneys, liver, and urine were harvested 40 min after injection of a low scaled molar dose of ${ }^{68} \mathrm{Ga}$-labeled IRDye800-tilmanocept (FL-tilmanocept) or ${ }^{68} \mathrm{Ga}$-labeled DTPA-IRDye800-galactosyl-dextran (FL-gal-dextran); injection of a high scaled dose of FL-tilmanocept; or coinjection of FL-tilmanocept and $5.0 \mathrm{nmol} / \mathrm{g}$ tilmanocept.

fitted with a high-resolution low-energy collimator. After a 25-min dynamic imaging study ${ }^{99 \mathrm{~m} T c}$-labeled Cy5-tilmanocept, $\sim 18.5 \mathrm{MBq}, 2.6$ nmol), both kidneys and the urinary bladder were excised, weighed, and assayed for radioactivity (100-200 keV) with a known dilution of the injectate. Regions of interest were drawn around a portion of the left kidney. Decay-corrected time-activity curves were generated and normalized to the $\% \mathrm{ID} / \mathrm{g}$.

\section{Statistical Analysis}

We used the Student $t$ test (JMP, version 9, 2010; SAS Institute) to calculate a 2-tailed test of statistical significance assuming equal variances. We considered a 2 -sided $P$ value of less than 0.05 to be statistically significant.

\section{RESULTS}

\section{PET Imaging of Healthy Rats: Demonstration of Glomerular CD206 Accumulation}

The biodistribution studies of the CD206-specific molecule IRDye800-tilmanocept and the negative control, IRDye800-DPTAgalactosyl-dextran, demonstrated receptor-mediated binding of tilmanocept within the kidney. Figure 2 summarizes these studies graphically. The low scaled molar dose studies demonstrated a statistically significant $(P<0.001)$ difference in renal uptake between tilmanocept and ${ }^{68} \mathrm{Ga}$-IRDye800-DTPA-galactosyl-dextran (FL-galDextran). The liver accumulation at $40 \mathrm{~min}$ after injection of the low-dose $(0.02 \mathrm{nmol} / \mathrm{g})$ tilmanocept and ${ }^{68} \mathrm{Ga}$-IRDye800-DTPAgalactosyl-dextran doses (FL-gal-Dextran, $0.02 \mathrm{nmol} / \mathrm{g}$ ) was significantly $(P<0.001)$ higher than the hepatic accumulation $(2.94 \pm$ $0.67 \mathrm{nmol} / \mathrm{g})$ of the high-dose $(0.10 \mathrm{nmol} / \mathrm{g})$ FL-tilmanocept studies. Saturation of the hepatic CD206 permitted more fluorescent-tilmanocept to reach the kidneys. The \% ID of urinary bladder and urine was significantly $(P=0.042)$ higher for the low-dose fluorescenttilmanocept studies $(14.2 \% \pm 7.8 \%)$ than for the low-dose fluorescent-galactosyl-dextran studies $(5.57 \% \pm 1.81 \%)$. Neither of the low-dose studies exhibited a statistically different urinary bladder accumulation compared with the high-dose tilmanocept $(8.16 \% \pm$ $4.42 \%)$.
PET dynamic imaging demonstrated receptor-mediated renal accumulation by ${ }^{68} \mathrm{Ga}$-IRDye800-tilmanocept. Four examples of renal time-activity curves are displayed in Figure 3. Renal accumulation by a high scaled molar dose (FL-tilmanocept) of ${ }^{68} \mathrm{Ga}-$ fluorescent-tilmanocept exhibited a longer time to peak ( $8.8 \mathrm{~min})$ and higher accumulation than an imaging study that used a low scaled molar dose (FL-tilmanocept) of fluorescent-tilmanocept. The liver accumulation for the same high scaled molar dose (Supplemental Fig. 1; supplemental materials are available at http:// jnm.snmjournals.org) FL-tilmanocept studies exhibited a nearly flat curve with a very low peak value $(31 \%)$. This permitted the kidneys to bind a greater fraction of the injectate. A negative control study (FL-gal-Dextran) exhibited negligible renal accumulation and high (81 \%ID) liver uptake (Supplemental Fig. 1); the highest kidney values occurred early after injection $(\sim 3.2 \mathrm{~min})$ and most probably were produced by activity within the renal blood pool. A coinjection (blocking) study (Fig. 3) exhibited low renal accumulation, which plateaued $(\sim 7.5 \% \mathrm{ID} / \mathrm{g})$ within $3 \mathrm{~min}$ of injection. The liver time-activity curve from this study also exhibited saturated binding (Supplemental Fig. 1). The shape of the kidney blocking curves differed significantly from the nonblocked 0.10 $\mathrm{nmol} / \mathrm{g}$ injections that did not contain a $5.0 \mathrm{nmol} / \mathrm{g}$ blocking dose of cold tilmanocept. The nonblocked curves exhibited a sustained renal accumulation for $10 \mathrm{~min}$ and achieved a $\% \mathrm{ID} / \mathrm{g}$ within each kidney that was double the plateau value of the blocked dose.

PET imaging demonstrated receptor-mediated accumulation of ${ }^{68} \mathrm{Ga}$-IRDye800-tilmanocept in the renal cortex. Figure $4 \mathrm{~A}$ is a ventral projection whole-body maximum-intensity-projection image (20-40 min after injection) and is representative of a high molar dose $(0.1 \mathrm{nmol} / \mathrm{g}){ }^{68} \mathrm{Ga}$-labeled-fluorescent-tilmanocept study, which exhibited diminished liver accumulation $(3.1 \% \mathrm{ID} / \mathrm{g})$ compared with the kidneys (12\% ID/g); the urinary bladder accumulated $14 \% \mathrm{ID}$. A PET maximum-intensity-projection whole-body image (Fig. 4B) from a low molar dose $(0.02 \mathrm{nmol} / \mathrm{g})$ study demonstrated a higher liver accumulation $(5.6 \% \mathrm{ID} / \mathrm{g})$ than the high scaled dose study. The kidneys accumulated $9.2 \% \mathrm{ID} / \mathrm{g}$ (Fig. 4B); the urinary bladder accumulated

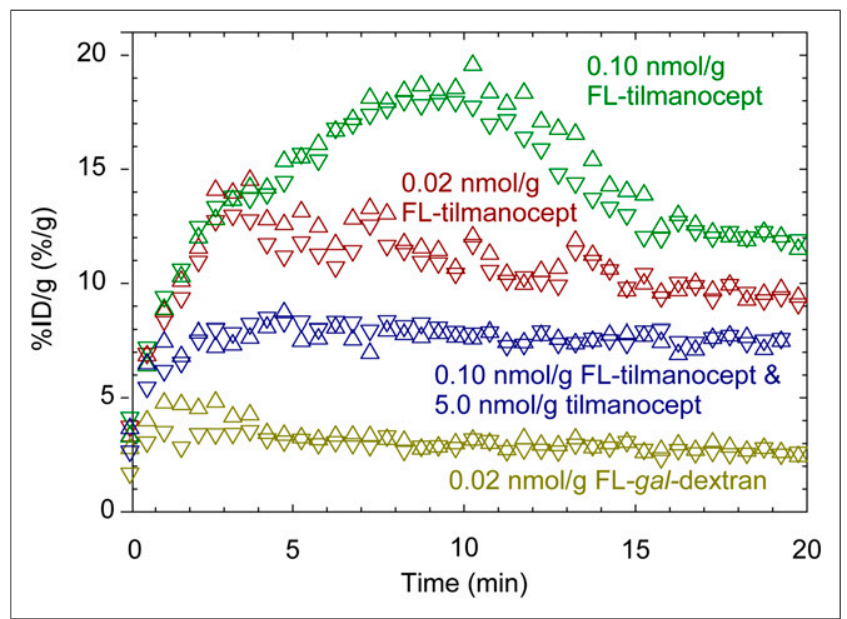

FIGURE 3. Renal cortex time-activity curves demonstrated receptormediated accumulation of tilmanocept. Left (upper triangles) and right (lower triangles) kidney time-activity curves displayed significantly different shapes after administration of low scaled molar dose of ${ }^{68} \mathrm{Ga}$ labeled IRDye800-tilmanocept (FL-tilmanocept) or ${ }^{68} \mathrm{Ga}$-labeled DTPAIRDye800-galactosyl-dextran (FL-gal-dextran); injection of high scaled dose of FL-tilmanocept; or coinjection of FL-tilmanocept and $5.0 \mathrm{nmol} / \mathrm{g}$ tilmanocept. 
$8.3 \%$ ID. The renal images of all $10{ }^{68} \mathrm{Ga}$-fluorescent-tilmanocept studies exhibited increased activity at the rim of the cortex. Figure $4 \mathrm{C}$ (the sum of 10 contiguous coronal cross-sections) clearly delineates activity within the renal cortex. Activity in the right and left cortex dominates the renal image. The dorsal aspect of the right and left lobes of the liver can be seen. A negative control study (Fig. 4D, maximum-intensity projection, whole-body, 20-40 min after injection) using ${ }^{68} \mathrm{Ga}$-IRDye 800 -galactosyl-dextran $(0.02 \mathrm{nmol} / \mathrm{g})$ exhibited significantly less radioactivity in the kidneys $(3.1 \% \mathrm{ID} / \mathrm{g})$.

\section{Immunohistochemistry: Demonstration of CD206 Targeting}

Histomicrographs of all 10 rat kidneys from the high dose ${ }^{68} \mathrm{Ga}-$ IRDye800-tilmancoept studies demonstrated tilmanocept binding to the CD206 receptor within the glomerulus. Figure 5 displays 4 histomicrographs from the same section using $\times 60$ magnification. Figure $5 \mathrm{~A}$ is a single channel (AlexaFluor647) demonstrating the distribution of CD206. Figure 5B is a single-channel (IRDye800CW, $\times 60)$ composite representing the distribution of IRDye800-tilmanocept. Figure 5C is a 2-channel (AlexaFluor488, IRDye800CW) overlay composite, which demonstrates CD206 distribution within the glomerular compartment defined by podocytes stained with Alexa-488. Lastly, Figure 5D is another 2-channel (AlexaFluor647, IRDye800CW) overlay composite that demonstrates tilmanocept and CD206 colocalization within 1 glomerulus.

Histomicrographs from the negative control studies demonstrated the specificity of tilmanocept for mesangial CD206 (Supplemental Fig. 2; supplemental materials are available at http:// jnm.snmjournals.org). Histomicrographs from healthy rat kidneys excised 40 min after injection of ${ }^{68} \mathrm{Ga}$-IRDye800-DTPA-galactosyl-dextran exhibited a very low presence of IRDye800. Histomicrographs using $\times 20$ magnification (Supplemental Fig. 3) also demonstrated colocalization of tilmanocept and CD206. These wider-field views displayed extraglomerular regions of IRDye800, which are consistent with filtered IRDye800-tilmanocept within the renal tubules.

\section{Y-Imaging of Mice: Demonstration of Sensitivity to Chronic Renal Disease}

Dynamic imaging of $d b / m$ and $d b / d b$ mice demonstrated kinetic sensitivity to chronic kidney disease. These experiments

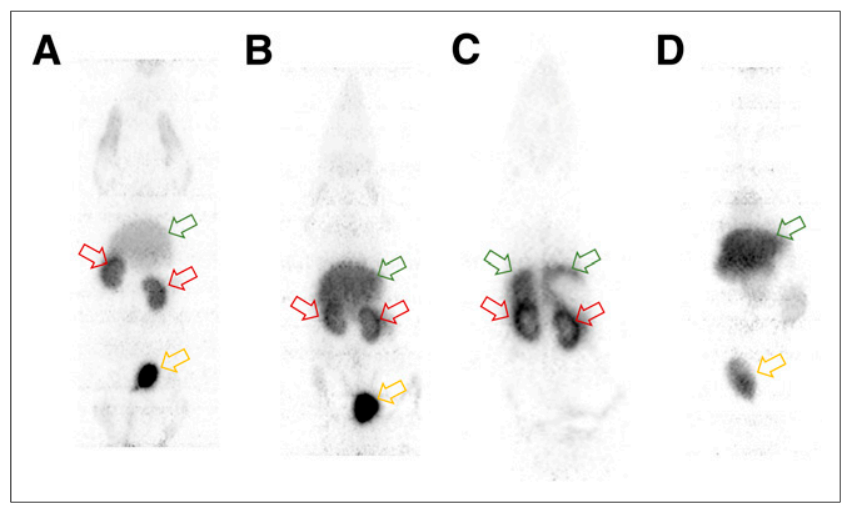

FIGURE 4. PET images demonstrated receptor-mediated accumulation of ${ }^{68} \mathrm{Ga}$-IRDye800-tilmanocept in renal cortex (red arrows). (A) Scaled high molar dose study demonstrated low liver accumulation (green arrow) compared with kidneys and urinary bladder (yellow arrow). (B) Low molar dose study demonstrated higher liver accumulation and lower kidney accumulation. (C) Coronal cross-sections more clearly delineate activity in renal cortex. (D) Negative control study (maximum-intensity projection) exhibited significantly less radioactivity in kidneys. All images were acquired 20-40 min after injection.

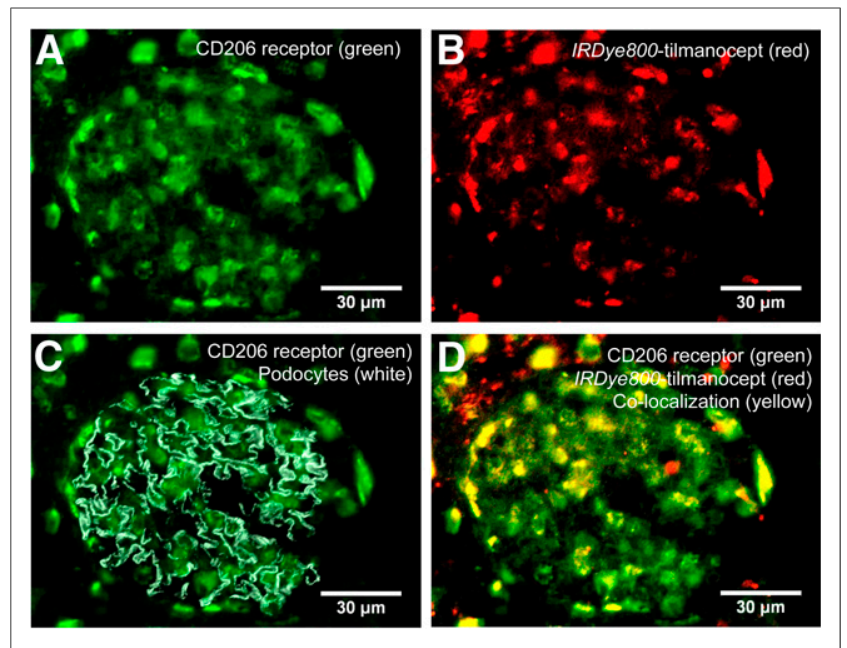

FIGURE 5. Histologic demonstration of tilmanocept binding to CD206 receptor within the mesangium. Four histomicrographs are shown from same section of healthy rat kidney excised 40 min after injection of IRDye800CW-tilmanocept. (A) Single channel representing distribution of CD206. (B) Single channel representing distribution of IRDye800-tilmanocept. (C) 2-channel composite, which demonstrates CD206 (green) distribution within glomerular compartment, which is defined by podocytes (gray). (D) 2-channel composite of CD206 (green) and IRDye800-tilmanocept (red) distributions (overlapping stains result in yellow).

are summarized in Figure 6. The average time at which the nondiabetic $d b / m$ renal curves peaked was significantly $(P<0.005)$ longer than the average time-to-peak value of the curves from the 4.9-wk-old $d b / d b$ diabetic mice, the 7.3-wk-old $d b / d b$ mice, and the 13.3 -wk-old $d b / d b$ mice. The renal $\% \mathrm{ID} / \mathrm{g}$ of ${ }^{99 \mathrm{~m} T c-l a b e l e d}$ Cy5-tilmanocept by nondiabetic $\mathrm{db} / \mathrm{m}$ mice far exceeded the renal accumulation of the diabetic $d b / d b$ mice at all 3 ages. The \% ID in the urine of the $d b / m$ mice was significantly lower $(P<0.03)$ than all age groups of the $d b / d b$ mice.

Figure 7A displays representative ${ }^{99 \mathrm{~m}}$ Tc-labeled Cy5-tilmanocept scaled time-activity curves from the left kidneys of diabetic and nondiabetic mice. The 4 curves illustrate the profound difference in shape between the time-activity curves of the nondiabetic and 3 diabetic mice. The nondiabetic $\mathrm{db} / \mathrm{m}$ mouse exhibited a renal uptake curve dominated by a single phase during the 25-min dynamic imaging study. The left kidney curve of the nondiabetic mouse peaked 22 min after injection at $5.5 \%$ of the injected dose. The urinary bladder with contents of the nondiabetic $\mathrm{db} / \mathrm{m}$ mice at $30 \mathrm{~min}$ after injection contained $1.1 \% \mathrm{ID}$. Figure 7B is an expanded view of Figure 7A that demonstrates increased renal accumulation of tilmanocept with increasing age. The curves from the 4.9-, 7.3-, and 13.3-wk-old diabetic kidneys peaked at $4.0 \% \mathrm{ID}$ (at $2.5 \mathrm{~min}$ ), $4.4 \% \mathrm{ID}$ (at $2.0 \mathrm{~min}$ ), and $4.9 \% \mathrm{ID}$ at $1.8 \mathrm{~min}$, respectively.

\section{DISCUSSION}

\section{PET Imaging of Healthy Rat: Demonstration of Glomerular CD206 Accumulation}

This work provided in vivo demonstration that CD206 is the molecular target when imaging the kidney with radiolabeled tilmanocept. We demonstrated a statistically significant loss of renal accumulation by the galactosyl-conjugate of dextran (21), which binds to the asialoglycoprotein receptor in the liver and does not bind to CD206 in the kidney. Using time-activity curves 


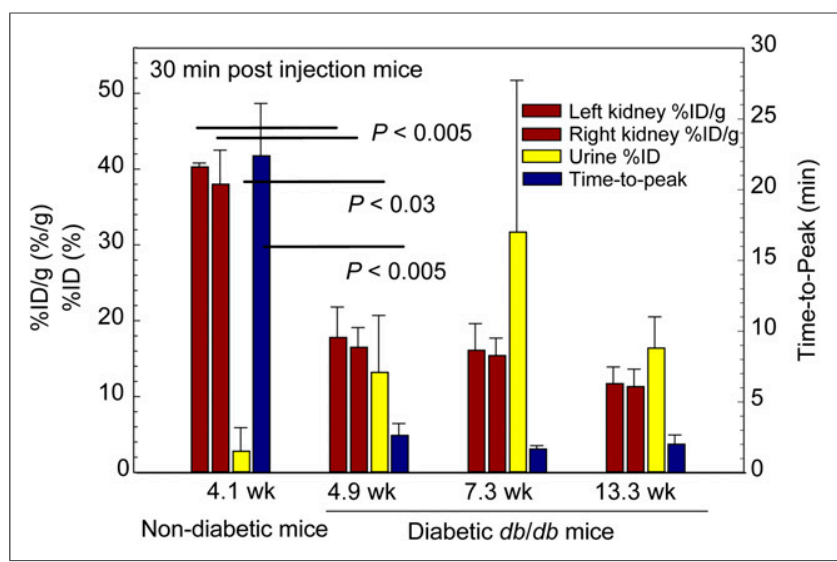

FIGURE 6. Studies in nondiabetic and diabetic mice demonstrated sensitivity to renal disease. Nondiabetic mice exhibited higher kidney $\% \mathrm{ID} / \mathrm{g}$ (red bars) and lower urine \%ID than diabetic mice at 4.9, 7.3, and $13.3 \mathrm{wk}$ of age. Time-activity curves peaked later in nondiabetic mice (blue bars) than diabetic mice.

at 2 different scaled molar doses, we demonstrated that the renal accumulation of ${ }^{68} \mathrm{Ga}$-labeled fluorescent-tilmanocept can be saturated, which is the hallmark of a receptor-mediated process (22). The standard method for demonstrating receptor-specific binding (23), a receptor blocking study, substantially diminished the renal accumulation of ${ }^{68} \mathrm{Ga}$-labeled tilmanocept.

\section{Immunohistochemistry: Demonstration of CD206 Targeting}

We demonstrated the histologic colocalization of IRDye800tilmanocept with a fluorescent marker for CD206. A substantial majority of the IRDye $800 \mathrm{CW}$ fluorescence was localized within the confines of the glomerulus, as defined by podocyte staining.

\section{y-Imaging of Mice: Demonstration of Sensitivity to Chronic Renal Disease}

The $d b / d b$ and $d b / m$ mouse imaging studies (Figs. 6 and 7) demonstrated kinetic sensitivity of tilmanocept to renal function in chronic renal disease. The $d b / d b$ mouse $(24,25)$ is a rodent model of genetic diabetes, which mirrors the progression of glomerular changes found in human diabetes $(4,26)$. At 8 wk of age, diabetic $d b / d b$ mice have the same serum creatinine levels and same mesangial matrix fraction as nondiabetic $\mathrm{db} / \mathrm{m}$ mice of the same age (27). At 12 and $16 \mathrm{wk}$ of age, the mesangial matrix fraction of $d b / d b$ mice expands 2- and 3.5-fold, respectively. This expansion was statistically significant when compared with the $d b /$ $d b$ mice at $4 \mathrm{wk}$ and the $d b / \mathrm{m}$ mice of the same age (27). The elevated ${ }^{99 \mathrm{~m}}$ Tc-tilmanocept renal and urine accumulation (Fig. 6) of the diabetic mice is consistent with hyperfiltration observed in $d b / d b$ mice and human diabetic nephropathy (26).

\section{The Mesangial Cell as an Alternative Molecular Imaging Target}

Intraglomerular mesangial cells offer a new cellular target for molecular imaging of the kidney. After delivery to the kidney by renal plasma flow (Fig. 8), an imaging agent can be passively ultrafiltered into the proximal tubule depending on its size and charge or extracted from the plasma by the mesangial cells. Both routes require passage through a highly porous and negatively charged (28) fenestrated capillary endothelium. Molecules that are passively filtered into the Bowman capsule traverse a dense network of glycoprotein and collagen known as the glomerular basement membrane, which is also negatively charged (29), followed by a negatively charged slit diaphragm between the podocytes. Molecules that approach the mesangial cells must traverse a fibrillary matrix, also known as the mesangial cell matrix.

An important physiologic function of mesangial cells is their active participation in the formation and maintenance of the mesangial cell matrix and the glomerular basement membrane (30). Thickening of the glomerular basement membrane and expansion of the mesangium are the major lesions of diabetic nephropathy leading to renal dysfunction in diabetes patients (31). These lesions occur early in the progression of the disease. Both the cellular and the matrix components of the mesangium increase in relative volume during diabetes (32), with a $57 \%$ increase in cellular volume and $185 \%$ increase in matrix volume. Other significant mesangial cell functions are the regulation of glomerular plasma flow, by their smooth muscle properties; the immunologic recognition and clearance of cellular debris within the mesangium; and the monitoring of mesangial glucose concentration.

\section{Clinical Significance}

Tilmanocept may offer a noninvasive alternative to renal biopsy for diagnosis of early glomerular disease. Many potential drug targets have emerged from this research, including the vasopressin $\mathrm{V} 1 \alpha$ receptor (33), the NF- $\mathrm{KB}$ receptor (34), and $\alpha_{\mathrm{V}} \beta_{3}$ (35). Agents that target these receptors are most effective during the early phases of inflammation, where they block the inflammatory response that leads to fibrosis. This strategy necessitates early recognition of disease. This has led to calls for universal renal biopsies in newly diagnosed diabetic patients to identify early treatable disease (36). Percutaneous biopsy is invasive, and the procedure alone carries a $7 \%-10 \%$ risk of minor complication such as bleeding or pain and a $0.1 \%-0.5 \%$ risk of a major complication requiring a nephrectomy or iatrogenic arteriovenous malformation (37). Given 1.7 million new cases of type 2 diabetes in 2012 (38), this would lead to $119,000-$ 170,000 patients with bleeding and pain after biopsy, and 1,700 to 7,500 patients
FIGURE 7. (A) $99 \mathrm{~m} T \mathrm{c}-$ labeled tilmanocept time-activity curves exhibited kinetic sensitivity to chronic kidney disease. Diabetic $d b / d b$ mice (green, $4.9 \mathrm{wk}$; yellow, $7.3 \mathrm{wk}$; red, $13.3 \mathrm{wk}$ ) peaked within $2 \mathrm{~min}$ after injection. Nondiabetic $\mathrm{db} / \mathrm{m}$ mouse (blue) peaked $18 \mathrm{~min}$ after injection. (B) Expanded view shows progressively faster accumulation with increasing age.

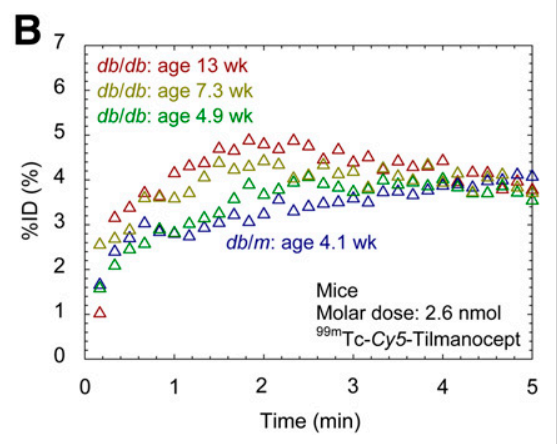

Mesangial Cell Molecular Imaging • Qin et al. 


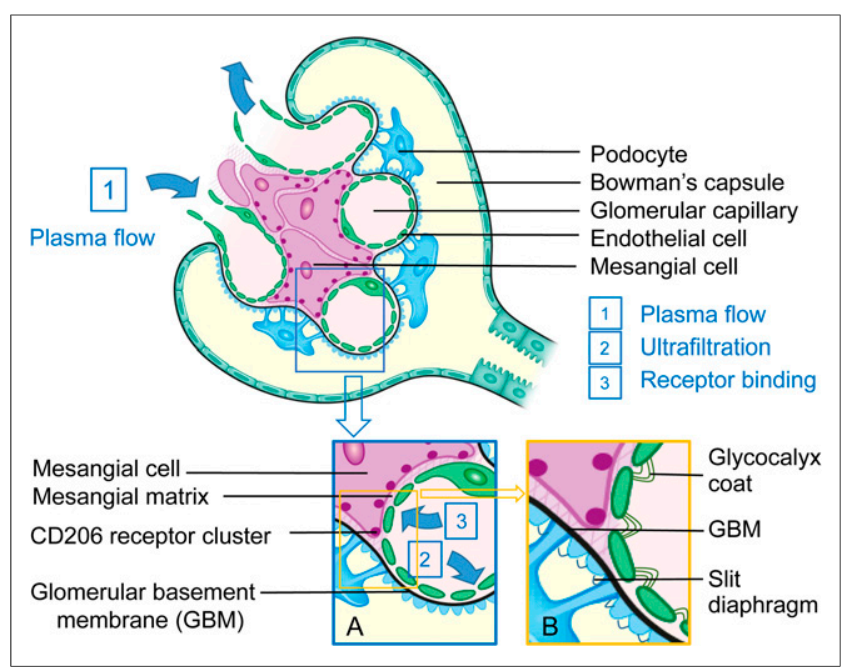

FIGURE 8. After delivery to capillary lumen by renal plasma flow (arrow 1), a molecule of radiolabeled IRDye800-tilmanocept can exit glomerulus via renal plasma flow, passively be ultrafiltered into Bowman capsule (inset $\mathrm{B}$, arrow 2), or passively be ultrafiltered into transverse mesangial cell matrix (inset $A$, arrow 3) and bind to CD206 receptor.

with major complications such as arteriovenous fistula or bleeding requiring partial nephrectomy (37). Finally, biopsy suffers from undersampling, as only 10 of 617,000 glomeruli per kidney are actually evaluated in the course of a renal biopsy (39).

\section{Optimization of the Molecular Imaging Agent}

Our next step toward the development of radiolabeled tilmanocept as a renal molecular imaging agent will be the design of a protocol that matches the clinical setting. Knowing that the cellular target within the kidney is responsible for mesangial matrix expansion, our design strategy will maximize the sensitivity of tilmanocept imaging to changes in both mesangial matrix permeability (Fig. 8), and receptor density. The following chemical features would make CD206-targeted molecular imaging highly sensitive to tilmanocept mesangial matrix permeability and receptor density: a molar dose of tilmanocept that does not saturate the receptor sites in healthy kidneys, a neutral charge distribution of the molecular imaging agent, and an imaging agent of moderately high molecular diameter. The last property will be used to eliminate ultrafiltration through the Bowman capsule, which will increase mesangial cell accumulation. A CD206-specific dextran with a molecular radius equal to albumin will not be filtered by the glomerular basement membrane but will still retain easy access through the mesangial matrix. The rat glomerulus has been studied by electron microscopy $(40,41)$, revealing an average mesangial matrix pore size that is 10 -fold larger than that of the glomerular basement membrane.

Our design hypothesis is that early mesangial matrix expansion will increase the permeability of tilmanocept through the mesangial matrix, which in turn will increase delivery of the molecular imaging agent to mesangial cells. This is consistent with the series of time-activity curves (Fig. 7A) from diabetic mice, where early time-points displayed (Fig. 7B) fast renal accumulation with increasing age. This hypothesis is based on measurements of collagen VI density, which decreases during mesangial matrix expansion (42). The increased delivery to the mesangial cell surface will expose more tilmanocept to the limited number of CD206 receptors. The result will be a timeactivity curve with a shape different from that of a healthy kidney (Fig.
3). This study used a high scaled molar dose, which produced a curve that exhibits receptor saturation distinguished by a slower and linear portion of the curve (5-10 min after injection) as it approaches its peak value.

For routine clinical applications, ${ }^{99 \mathrm{~m}} \mathrm{Tc}$ is the nuclide of choice. The typical imaging protocol could consist of a $370-\mathrm{MBq}$ dose of ${ }^{99} \mathrm{~m}$ Tc-tilmanocept administered intravenously followed by a 20 -min dynamic imaging study; the patient would lie supine with both kidneys within the field of view of an anterior-facing camera head, with the second camera head acquiring a posterior view. The dynamic study would provide a quantitative basis to assess the glomerular function of each kidney.

Clinical research could use PET/MRI or PET/CT with ${ }^{68} \mathrm{Ga}$. PET imaging with motion correction via simultaneous MRI acquisition could provide regional estimates of mesangial cell function. Although it has been demonstrated (5) that mesangial expansion is characterized by a 3-fold increase in mesangial matrix over mesangial cell volume, dynamic PET/MRI using higher scaled molar doses of ${ }^{68} \mathrm{Ga}$-tilmanocept could provide independent measurements of permeability-surface area of the mesangial matrix, as well as receptor density. The latter measurement should be directly proportional to mesangial cell density. An example of this approach is hepatocellular imaging of ${ }^{99 \mathrm{~m}} \mathrm{Tc}$-galactosyl-neoglycoalbumin $(43,44)$, where kinetic modeling demonstrated a linear relationship between receptor amount and viable hepatocellular mass within the entire liver (44).

Imaging of specific molecular targets, such as CD206 within the glomerulus and the organic anion transporter 1 of proximal tubules $(45,46)$, represents a complementary strategy for the noninvasive measurement of renal function. Both targets are energy-dependent transporters of physiologically significant molecules at 2 critical cell types that serve different functions within the kidney. The radiopharmaceuticals ${ }^{99 \mathrm{~m} T c-D T P A}$ and ${ }^{99 \mathrm{~m}} \mathrm{Tc}-$ mercaptoacetyltriglycine have specific roles but are not accepted (47) as the primary indication for glomerular filtration rate or renal blood flow. Most importantly, the detection of blood flow, filtration, and structural abnormalities, such as cortical atrophy and tumors, is unable to determine the early stages of chronic kidney disease, such as diabetes, or immune complex diseases, such as lupus or IgA nephropathy. Currently, there are no biomarkers for the early detection of diabetic nephropathy (48) and immune complex diseases of the kidney (49). Consequently, these diseases are screened by GFR estimates from serum creatinine measurements and urine microalbumin concentrations (50), both of which are insensitive in the first decade of type 1 and type 2 diabetes $(31,51,52)$.

\section{CONCLUSION}

This study demonstrated receptor-mediated binding to the glomerular mesangial cells and kinetic sensitivity of tilmanocept to chronic renal disease. Given the early role of mesangial cells during the progression of chronic kidney disease, PET or SPECT renal imaging of CD206 may provide a noninvasive and highly sensitive quantitative assessment of glomerular function based on specific physiologic and cellular components that are central to the healthy kidney. A SPECT/CT or PET/CT protocol that accurately staged early diabetic kidney disease may help to establish a precise baseline for frequent surveillance by a serum or urine biomarker.

\section{DISCLOSURE}

David Vera is the inventor of tilmanocept. No other potential conflict of interest relevant to this article was reported. 


\section{ACKNOWLEDGMENTS}

We thank Dr. William C. Eckelman and Dr. Sushrut Waikar for their helpful discussions. We also thank the staffs of the In Vivo Imaging Shared Resource and the Microscope Shared Resource at the University of California, San Diego, Moores Cancer Center. This work was funded in part by the NCI In Vivo Cancer and Molecular Imaging Program (P50 CA128346). Dr. Jahromi was supported by NIH T32-4T32EB005970. This work was presented to the NIDDK Renal Imaging Workshop, July 12-13, 2018, Bethesda, MD; we acknowledge the many helpful comments from the workshop participants.

\section{KEY POINTS}

QUESTION: The objective of this study was to demonstrate receptor-mediated binding of tilmanocept to CD206 within the kidney and provide evidence of kinetic sensitivity of kidney timeactivity data to chronic renal function.

PERTINENT FINDINGS: This study demonstrated receptor-mediated binding of tilmanocept to rodent glomerular mesangial cells and kinetic sensitivity of tilmanocept to a murine model of diabetic nephropathy.

IMPLICATIONS FOR PATIENT CARE: Renal imaging of radiolabeled tilmanocept may provide the unmet need for a predictive tool of kidney function during the first decade of disease, when there is silent loss of glomerular function.

\section{REFERENCES}

1. Ogurtsova K, da Rocha Fernandes JD, Huang Y, et al. IDF diabetes atlas: global estimates for the prevalence of diabetes for 2015 and 2040. Diabetes Res Clin Pract. 2017;128:40-50.

2. KDOQI. KDOQI clinical practice guidelines and clinical practice recommendation for diabetes and chronic kidney disease. Am J Kidney Dis. 2007;49(suppl): S12-S1544.

3. Alicic RZ, Rooney MT, Tuttle KR. Diabetic kidney disease: challenges, progress, and possibilities. Clin J Am Soc Nephrol. 2017;12:2032-2045.

4. Thomas MC, Brownlee M, Susztak K, et al. Diabetic kidney disease. Nat Rev Dis Primers. 2015;1:15018.

5. Steffes MW, Osterby R, Chavers B, Mauer SM. Mesangial expansion as a central mechanism for loss of kidney function in diabetic patients. Diabetes. 1989;38: 1077-1081.

6. Katz A, Caramori ML, Sisson-Ross S, Groppoli T, Basgen JM, Mauer M. An increase in the cell component of the cortical interstitium antedates interstitial fibrosis in type 1 diabetic patients. Kidney Int. 2002;61:2058-2066.

7. Mauer SM, Steffes MW, Ellis EN, Sutherland DE, Brown DM, Goetz FC. Structural-functional relationships in diabetic nephropathy. J Clin Invest. 1984;74: 1143-1155.

8. Caramori ML, Kim Y, Huang C, et al. Cellular basis of diabetic nephropathy: 1 . Study design and renal structural-functional relationships in patients with longstanding type 1 diabetes. Diabetes. 2002;51:506-513.

9. Fioretto P, Steffes MW, Sutherland DE, Goetz FC, Mauer M. Reversal of lesions of diabetic nephropathy after pancreas transplantation. N Engl J Med. 1998;339: 69-75.

10. Sabbisetti V, Bonventre JV. Biomarkers in acute and chronic kidney diseases. In: Tall MW, Chertow GM, Marsden PA, Skorecki K, Yu ASL, Brenner BM, eds. Brenner \& Rector's The Kidney. 9th ed. Philadelphia, PA: Elsevier Saunders; 2012: 1016-1042.

11. Linehan SA, Martinez-Pomares L, Stahl PD, Gordon S. Mannose receptor and its putative ligands in normal murine lymphoid and nonlymphoid organs: in situ expression of mannose receptor by selected macrophages, endothelial cells, perivascular microglia, and mesangial cells, but not dendritic cells. J Exp Med. 1999;189: 1961-1972.

12. Vera DR, Wallace AM, Hoh CK, Mattrey RF. A synthetic macromolecule for sentinel node detection: ${ }^{99 m}$ Tc-DTPA-mannosyl-dextran. J Nucl Med. 2001;42:951-959.

13. Sirlin CB, Vera DR, Corbeil JA, Caballero MB, Buxton RB, Mattrey RF. Gadolinium-DTPA-dextran: a macromolecular MR blood pool contrast agent. Acad Radiol. 2004;11:1361-1369.
14. Vera DR, Krohn KA, Stadalnik RC, Scheibe PO. Tc-99m galactosyl-neoglycoalbumin: in vitro characterization of receptor-mediated binding. J Nucl Med. 1984;25: 779-787.

15. Stroup SP, Kane CJ, Farchshchi-Heydari S, et al. Preoperative sentinel lymph node mapping of the prostate using PET/CT fusion imaging and Ga-68-labeled tilmanocept in a dog model. Clin Exp Metastasis. 2012;29:673-680.

16. Emerson DK, Limmer KK, Hall DJ, et al. A receptor-targeted fluorescent radiopharmaceutical for multi-reporter sentinel lymph node imaging. Radiology. 2012;265: 186-193.

17. Vera DR, Stadalnik RC, Krohn KA. [Tc-99m]galactosyl-neoglycoalbumin: preparation and preclinical studies. J Nucl Med. 1985;26:1157-1167.

18. Qin Z, Hall DJ, Liss MA, et al. Optimization via specific fluorescence brightness of a receptor-targeted probe for optical imaging and positron emission tomography of sentinel lymph nodes. J Biomed Opt. 2013;18:101315.

19. Qin Z, Hoh CK, Hall DJ, Liss MA, Kane CJ, Vera DR. Radiolabeling and quality control of fluorescent-labeled tilmanocept for tri-model sentinel lymph node mapping. Nucl Med Biol. 2015;42:917-922.

20. Wallis JW, Miller TR. Three-dimensional display in nuclear medicine and radiology. J Nucl Med. 1991;32:534-546.

21. Vera DR, Hall DJ, Hoh C, Gallant P, McIntosh L, Mattrey R. Cy5.5-DTPAgalactosyl-dextran: a fluorescent probe for in vivo measurement of receptor biochemistry. Nucl Med Biol. 2005;32:687-693.

22. Vera DR, Krohn KA, Stadalnik RC, Scheibe PO. [Tc-99m]galactosyl-neoglycoalbumin: in vivo characterization of receptor-mediated binding to hepatocytes. Radiology. 1984;151:191-196.

23. Eckelman WC, Frank JA, Brechbiel M. Theory and practice of imaging saturable binding sites. Invest Radiol. 2002;37:101-106.

24. Hummel KP, Dickie MM, Coleman DL. Diabetes, a new mutation in the mouse. Science. 1966;153:1127-1128.

25. Cohen MP, Clements RS, Hud E, Cohen JA, Ziyadeh FN. Evolution of renal function abnormalities in the $\mathrm{db} / \mathrm{db}$ mouse that parallels the development of human diabetic nephropathy. Exp Nephrol. 1996;4:166-171.

26. Sharma K, McCue P, Dunn SR. Diabetic kidney disease in the db/db mouse. Am J Physiol Renal Physiol. 2003;284:F1138-F1144.

27. Cohen MP, Lautenslager GT, Shearman CW. Increased urinary type IV collagen marks the development of glomerular pathology in diabetic dd/db mice. Metabolism. 2001;50:1435-1440.

28. Sörensson J, Bjornson A, Ohlson M, Ballermann BJ, Haraldsson B. Synthesis of sulfated proteoglycans by bovine glomerular endothelial cells in culture. Am J Physiol Renal Physiol. 2003;284:F373-F380.

29. Kanwar YS, Farquhar MG. Presence of heparan sulfate in the glomerular basement membrane. Proc Natl Acad Sci USA. 1979;76:1303-1307.

30. Schlondorff $D$. The glomerular mesangial cell: an expanding role for a specialized pericyte. FASEB J. 1987;1:272-281.

31. Parving H-H, Mauer M, Fiorette P, Rossing P, Ritz E. Diabetic nephropathy. In: Tall MW, Chertow GM, Marsden PA, Skorecki K, Yu ASL, Brenner BM, eds. Brenner \& Rector's The Kidney. 9th ed. Philadelphia, PA: Elsevier Sanders; 2012:1417.

32. Steffes MW, Bilous RW, Sutherland DE, Mauer SM. Cell and matrix components of the glomerular mesangium in type I diabetes. Diabetes. 1992;41:679684.

33. Windt WA, Tahara A, Kluppel AC, de Zeeuw D, Henning RH, van Dokkum RP. Early, but not late therapy with a vasopressin V1a-antagonist ameliorates the development of renal damage after 5/6 nephrectomy. J Renin Angiotensin Aldosterone Syst. 2006;7:217-224.

34. Oguiza A, Recio C, Lazaro I, et al. Peptide-based inhibition of IкB kinase/nuclear factor- $\kappa \mathrm{B}$ pathway protects against diabetes-associated nephropathy and atherosclerosis in a mouse model of type 1 diabetes. Diabetologia. 2015;58:1656-1667.

35. Maile LA, Busby WH, Gollahon KA, et al. Blocking ligand occupancy of the alphaVbeta3 integrin inhibits the development of nephropathy in diabetic pigs. Endocrinology. 2014;155:4665-4675.

36. Gonzalez Suarez ML, Thomas DB, Barisoni L, Fornoni A. Diabetic nephropathy: is it time yet for routine kidney biopsy? World J Diabetes. 2013;4:245255.

37. Brachemi S, Bollee G. Renal biopsy practice: what is the gold standard? World J Nephrol. 2014;3:287-294.

38. Centers for Disease Control and Prevention. Estimates of Diabetes and Its Burden in the United States, 2014. Atlanta, GA: National Center for Chronic Disease Prevention and Health Promotion; 2014.

39. Nyengaard JR, Bendtsen TF. Glomerular number and size in relation to age, kidney weight, and body surface in normal man. Anat Rec. 1992;232:194201.

40. Latta $\mathrm{H}$, Fligiel S. Mesangial fenestrations, sieving, filtration, and flow. Lab Invest. 1985;52:591-598. 
41. Takami H, Naramoto A, Nakazawa K, Shigematsu H, Ohno S. Ultrastructure of glomerular mesangial matrix by quick-freeze and deep-etch methods. Kidney Int. 1990;38:1211-1215.

42. Moriya T, Groppoli TJ, Kim Y, Mauer M. Quantitative immunoelectron microscopy of type VI collagen in glomeruli in type I diabetic patients. Kidney Int. 2001;59: 317-323.

43. Stadalnik RC, Kudo M, Eckelman WC, Vera DR. In vivo functional imaging using receptor-binding radiopharmaceuticals: technetium-99m-galactosyl-neoglycoalbumin as a model. Invest Radiol. 1993;28:64-70.

44. Miki K, Kubota K, Inoue T, Vera DR, Makuuchi M. Receptor measurements via TcGSA kinetic modeling are proportional to functional hepatocellular mass. $J$ Nucl Med. 2001;42:733-737.

45. Lipowska M, Klenc J, Jarkas N, Marzilli LG, Taylor AT. Monoanionic ${ }^{99 m} \mathrm{Tc}-$ tricarbonyl-aminopolycarboxylate complexes with uncharged pendant groups: radiosynthesis and evaluation as potential renal tubular tracers. Nucl Med Biol. 2017;47:48-55.
46. Lipowska M, Jarkas $\mathrm{N}$, Voll RJ, et al. $\operatorname{Re}(\mathrm{CO})_{3}\left(\left[{ }^{18} \mathrm{~F}\right] \mathrm{FEDA}\right)$, a novel ${ }^{18} \mathrm{~F}$ PET renal tracer: radiosynthesis and preclinical evaluation. Nucl Med Biol. 2018;58: 42-50.

47. Blaufox MD. Renal background correction and measurement of split renal function: the challenge-editorial comment: EJNM-D-15-00322, M Donald Blaufox, MD, PhD. Eur J Nucl Med Mol Imaging. 2016;43:548-549.

48. Currie G, McKay G, Delles C. Biomarkers in diabetic nephropathy: present and future. World J Diabetes. 2014;5:763-776.

49. De Vriese AS, Fervenza FC. Con: biomarkers in glomerular diseases-putting the cart before the wheel? Nephrol Dial Transplant. 2015;30:885-890.

50. Snyder S, Pendergraph B. Detection and evaluation of chronic kidney disease. Am Fam Physician. 2005;72:1723-1732.

51. McGowan T, McCue P, Sharma K. Diabetic nephropathy. Clin Lab Med. 2001;21: 111-146.

52. Saito R, Rocanin-Arjo A, You YH, et al. Systems biology analysis reveals role of MDM2 in diabetic nephropathy. JCI Insight. 2016;1:e87877. 